\title{
HIGH RESOLUTION FOURIER TRANSFORM SPECTROSCOPY OF LASER INDUCED FLUORESCENCE IN THE LITHIUM DIMER
}

\author{
C. LINTON, R. BACIS*, P. CROZET*, F. MARTIN*, A.J. ROSS* and \\ J. VERGES** \\ Physics Department, University of New Brunswick, P.O. Box 4400, Fredericton, \\ New Brunswick E3B 5A3, Canada. \\ * Laboratoire de Spectrométrie Ionique et Moléculaire, Bât. 205, Université Lyon I, \\ F-69622 Villeurbanne cedex, France \\ **Laboratoire Aimé Cotton (CNRS II), Université de Paris-Sud (Paris IX), F-91405 Orsay, France
}

\begin{abstract}
Several new states of $\mathrm{Li}_{2}$ have been observed in fluorescence, excited by $\mathrm{Ar}^{+}, \mathrm{Kr}^{+}$and cw dye lasers. Results from the analysis of high resolution Fourier transform spectra are reviewed, and spectroscopic constants are compared with ab initio predictions.
\end{abstract}

\section{Introduction}

The lithium dimer is one of the most extensively studied diatomic molecules, both experimentally and theoretically. With the advent of large scale computational facilities, sophisticated all-electron calculations became possible for light molecules such as $\mathrm{Li}_{2}$, and high quality ab initio potential energy curves have been computed for the valence states and for many of the Rydberg states of $\mathrm{Li}_{2}, / 1,2,3 /$. Experimental data have been essential in evaluating the theoretical predictions concerning excited electronic states, and various techniques have been employed to extend the data base of spectroscopic information available. The different methods used (e.g. excitation spectroscopy, dispersed fluorescence, perturbation facilitated optical-optical double resonance (PFOODR) among others) complement each other, and together give a solid description of the valence states of $\mathrm{Li}_{2}$. We present here the contribution from laser induced fluorescence recorded by Fourier transform spectrometry over the past five years. These experiments have led to the first observation of the (2) ${ }^{1} \Sigma_{g}^{+}$ $/ 4,5 /$ and $(2)^{1} \Sigma_{u}^{+} / 6 /$ states and the $(1)^{1} \Pi_{\mathrm{g}}$ state in ${ }^{6} \mathrm{Li}_{2} / 7 /$, and have also produced extensive data on the triplet systems $(1)^{3} \Sigma_{g}^{+}-a^{3} \Sigma_{u}^{+} / 8,9 /$ and $(1)^{3} \Delta_{g}-b^{3} \Pi_{u} / 10 /$.

\section{Laser induced fluorescence in the lithium dimer.}

The great advantage of laser induced fluorescence lies in its selectivity. Direct fluorescence occurs from a very small number of rovibrational levels of a given excited electronic state, giving a simple, uncrowded spectrum. In the absence of relaxation, the technique gives very little information about the upper state (sometimes only a single term energy $\mathrm{T}_{\mathrm{v}, \mathrm{J}}$ ) but may cover a limited number of rotational levels in many vibrational levels of several lower lying electronic states. The information contained in a spectrum may be enhanced if the lifetime of the upper state is long enough that collisions populate rotational (and occasionally vibrational) energy levels close to the single level pumped by the laser, in this case, series of relaxation lines surround the principal transitions. More rarely, electronic energy transfer occurs as a result of collisions, populating electronic states which are not accessible via laser excitation from the ground state. In $\mathrm{Li}_{2}$, we have observed multiple examples of both direct and collisionally induced fluorescence. 
We form $\mathrm{Li}_{2}$ in a heatpipe oven at $1100 \mathrm{~K}$, using about 10 Torr argon as a buffer gas. The lithium vapour is excited axially by a laser beam, which is either a fixed frequency from an argon or krypton ion laser, or a c.w. dye laser - usually in monomode operation. Backwards fluorescence is collected on a pierced mirror, and focused with a lens on to the entrance aperture of a Fourier transform spectrometer. The spectra are always Doppler limited in resolution.

Results.

The electronic transitions we have recorded in fluorescence are indicated in Figure 1 below.

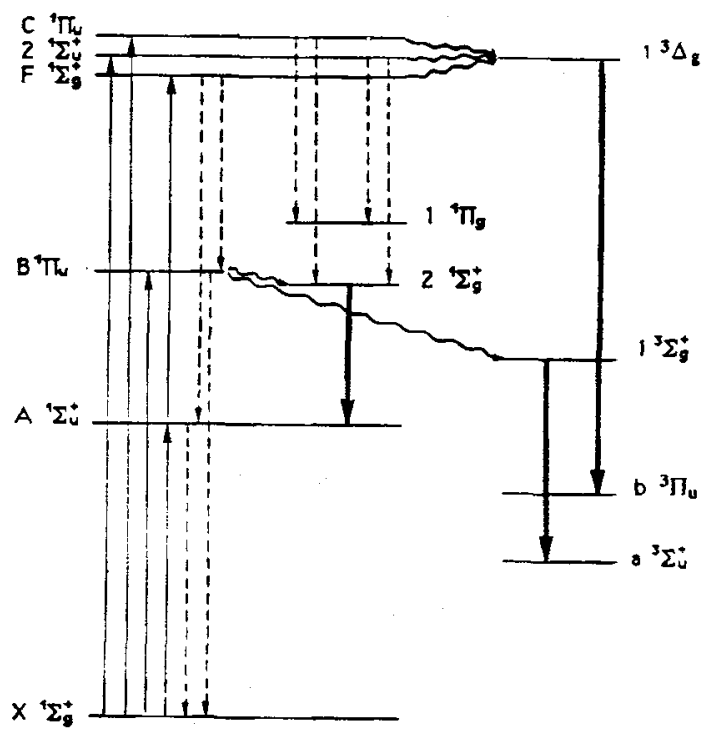

Figure 1 .

Laser induced fluorescence in $\mathrm{Li}_{2}$

Key

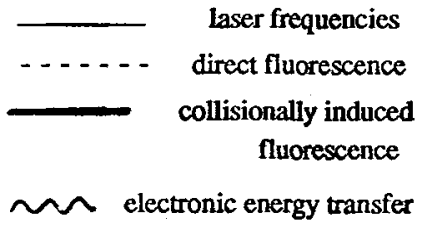

Using uv lines from an argon or krypton ion laser to excite the $C^{1} \Pi_{u}$ state and the (2) ${ }^{1} \Sigma_{u}^{+}$ (double minimum) state of $\mathrm{Li}_{2}$, we observed in the infrared direct fluorescence to (2) ${ }^{1} \Sigma_{\mathrm{g}}^{+}$and to (1) ${ }^{4} \pi_{g}$. The latter was the first experimental observation of the (1) ${ }^{1} \Pi_{\mathrm{g}}$ state in ${ }^{6} \mathrm{Li}_{2}$, and allowed us to measure the dissociation energy of this state, and to study, in detail, interactions between (2) ${ }^{1} \Sigma_{g}^{+}$and (1) ${ }^{1} \Pi_{g}$. Figure 2 shows a progression of doublets converging to the dissociation limit $\mathrm{Li}(2 \mathrm{~s}){ }^{2} \mathrm{~S}_{1 / 2}+$ Li (2p) ${ }^{2} P_{3 / 2}$. The same laser lines produced a collisionally induced spectrum, $(1)^{3} \Delta_{g}-b^{3} \Pi_{u}$ in the visible region, see below.

Figure 2. $C^{1} \Pi_{u}-(1){ }^{1} \Pi_{g}$ fluorescence in ${ }^{6} \mathrm{Li}_{2}$.

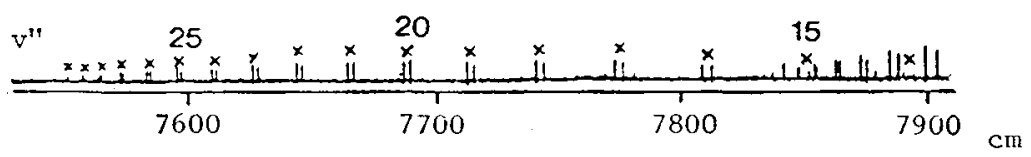

Exciting $\mathrm{Li}_{2}$ with $\mathrm{Ar}^{+} 4880$ and $4765 \AA$ gave, alongside the visible $\mathrm{B}-\mathrm{X}$ fluorescence, a collisionally induced spectrum of the $(2)^{1} \Sigma_{g}^{+}-A^{1} \Sigma_{u}^{+}$system in the IR. The upper state showed unusual vibrational distribution as a result of the mixing of ionic and neutral atomic character in the potential curve. The data from ${ }^{6} \mathrm{Li}_{2}$ and ${ }^{7} \mathrm{Li}_{2}$ for the $(2)^{4} \Sigma_{9}^{+}$state were isotopically consistent. The most intense transitions were those in which the $(2)^{1} \Sigma_{g}^{+}$levels had the same $\mathrm{J}$ and lay close in energy to the excited level in $\mathrm{B}^{1} \Pi_{u}$. 
A second collisionally induced transition following initial excitation to B ${ }^{1} \Pi_{u}$ accessed the triplet manifold of Li2. The upper state populated by collision is (1) ${ }^{3} \Sigma_{g}^{+}$, which lies $4000 \mathrm{~cm}-1$ below the level pumped directly in $B^{4} \Pi_{u}$. The transfer appears to be non selective in $\Delta v$ and $\Delta J$. From high resolution spectra of (1) ${ }^{3} \Sigma_{g}^{+} \rightarrow a{ }^{3} \Sigma_{u}^{+}$, we constructed an accurate potential energy curve for the weakly bound $a^{3} \Sigma_{u}^{+}$state.

An illustration of the different types of spectra obtained is given by a single colour double resonance experiment. We found that when we used a tunable dye laser, operating monomole with DCM dye, to excite lithium vapour, a few particular frequencies produced a bright green fluorescence. Each of these frequencies was resonant simultaneously with transitions in the $\mathrm{A}-\mathrm{X}$ and $\mathrm{F}-\mathrm{A}$ systems. The double resonant excitation of $F^{4} \Sigma_{g}^{+}$is followed by collisional transfer to (1) ${ }^{3} \Delta_{g}$ and we observe the (1) ${ }^{3} \Delta_{g}-b^{3} \Pi_{u}$ transition in the green region. Figure 3 shows spectra obtained with the laser tuned to $15047.53 \mathrm{~cm}^{-1}$.

\section{Figure 3}

The laser pumps $P(10)$ 4-0 in the $\mathrm{A}^{1} \Sigma_{u}^{+} \leftarrow X^{1} \Sigma_{g}^{+}$transition, giving rise to the direct $\mathrm{A} \rightarrow \mathrm{X}$ fluorescence in spectrum $a$. The main doublets are accompanied by extensive rotational relaxation in this case. This laser line is also resonant with $\mathrm{P}(9) \quad 1-4$ in the $\mathrm{F}^{1} \Sigma_{\mathrm{g}}^{+} \leftarrow \mathrm{A}^{1} \Sigma_{u}^{+}$ transition, and again the direct fluorescence, $F \rightarrow A$ is observed see spectrum $b$. The rotational relaxation is much weaker in this system. Spectrum $c$ shows part of the collisionally induced (1) ${ }^{3} \Delta_{g}-b$ ${ }^{3} \Pi_{\mathrm{u}}$ system. Only bands with $\mathrm{v}=0$ in the (1) ${ }^{3} \Delta_{\mathrm{g}}$ state are observed, but the rotational selectivity is reduced.

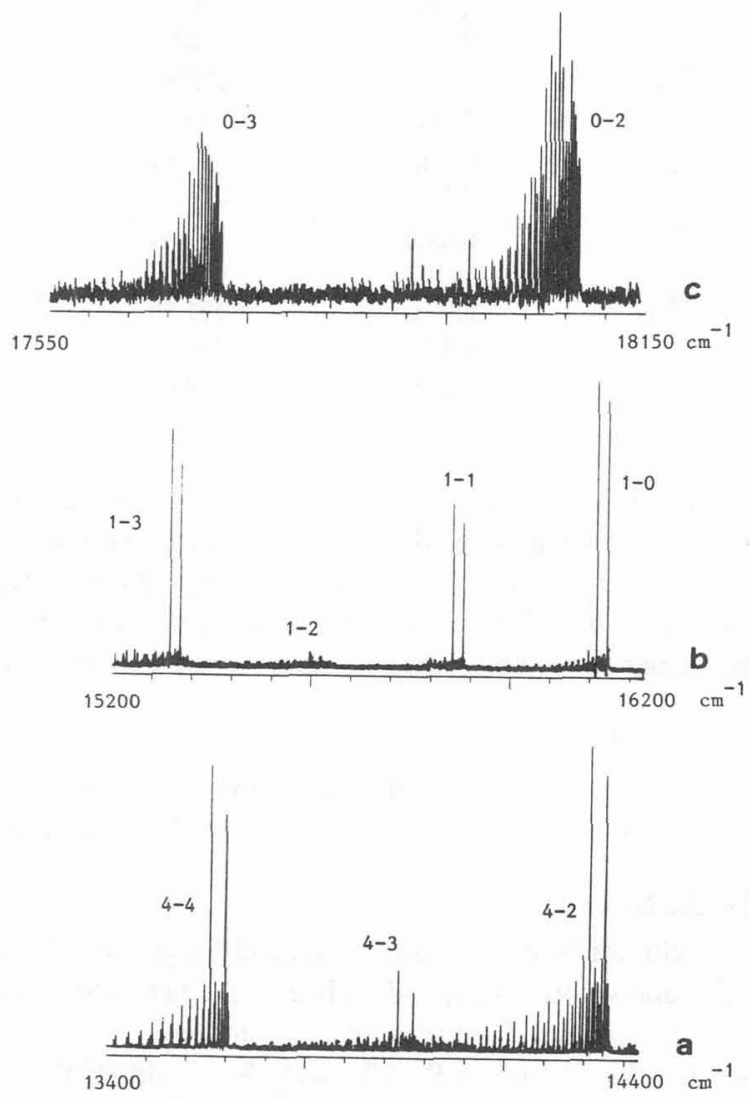

Combining the high resolution data on the (1) ${ }^{3} \Delta_{g}-b^{3} \Pi_{u}$ sytem obtained in collisionally induced fluorescence following argon, krypton or dye laser excitation, we find that collisional transfer from $C^{1} \Pi_{u},(2)^{1} \Sigma_{u}^{+}$and $F^{1} \Sigma_{g}^{+}$to (1) ${ }^{3} \Delta_{g}$, tends to occur to the nearest rotational level allowing the quantum number $J$ to be conserved, and always conserves the a,s symmetry of the rotational levels. We have studied line broadening and shifts caused by predissociation of $b^{3} \Pi_{u}$ by a ${ }^{3} \Sigma_{u}^{+}$and our measurements are in good agreement with $a b$ initio calculations, $/ 1 /$. We have also observed perturbations in (1) ${ }^{3} \Delta_{g}$ due to interactions with (2) ${ }^{3} \Pi_{g}$. 


\section{Conclusions}

We have observed several new electronic states of $\mathrm{Li}_{2}$ and extended observations of others. A summary is given in the table of constants below, with ab initio values quoted for comparison.

Table 1. Spectroscopic constants for ${ }^{6} \mathrm{Li}_{2}$ in $\mathrm{cm}^{-1}$.

Experimental values (boldface) with ab initio values from $/ 1$ / beneath (*indicates ab initio values from $/ 3$ ).

\begin{tabular}{|c|c|c|c|c|c|}
\hline State & $T_{e}$ & $\omega_{\mathrm{e}}$ & $\omega_{\mathrm{e}} \mathrm{X}_{\mathrm{e}}$ & $B_{e}$ & $D_{e}$ \\
\hline $2^{4} \Sigma_{g}^{+}$ & $\begin{array}{l}\text { 20101.58 } \\
20128\end{array}$ & $\begin{array}{l}138.92 \\
139.96\end{array}$ & $\begin{array}{l}-0.519 \\
-0.49\end{array}$ & $\begin{array}{l}0.42037 \\
0.419\end{array}$ & \\
\hline $1^{4} \Pi_{g}$ & $\begin{array}{l}21998.80 \\
21998\end{array}$ & $\begin{array}{l}100.06 \\
99.20\end{array}$ & $\begin{array}{l}1.966 \\
1.894\end{array}$ & $\begin{array}{l}0.3398 \\
0.3379\end{array}$ & $\begin{array}{l}1422.03 \\
1406 .\end{array}$ \\
\hline $2^{1} \Sigma_{u}^{+}$ & $\begin{array}{l}30100.26 \\
30077\end{array}$ & $\begin{array}{l}\mathbf{2 7 9 . 8 6} \\
279.93\end{array}$ & $\begin{array}{l}2.453 \\
1.83\end{array}$ & $\begin{array}{l}0.58401 \\
0.5874\end{array}$ & \\
\hline $1^{3} \Sigma_{g}^{+}$ & $\begin{array}{l}16328.8 \\
16333\end{array}$ & $\begin{array}{l}271.61 \\
272.4\end{array}$ & $\begin{array}{l}2.736 \\
2.803\end{array}$ & $\begin{array}{l}0.5954 \\
0.5958\end{array}$ & \\
\hline$a^{3} \Sigma_{u}^{+}$ & $\begin{array}{l}8183.8 \\
8194.8\end{array}$ & $\begin{array}{l}70.309 \\
68.83\end{array}$ & $\begin{array}{l}3.79 \\
3.74\end{array}$ & $\begin{array}{l}0.3323 \\
0.3199\end{array}$ & $\begin{array}{l}333 \\
322\end{array}$ \\
\hline $1^{3} \Delta_{g}$ & $\begin{array}{l}\mathbf{3 0 0 9 2} \\
30309 *\end{array}$ & $\begin{array}{l}\mathbf{3 0 2 . 5 9} \\
304.67^{*}\end{array}$ & 2.047 & $\begin{array}{l}\mathbf{0 . 6 1 6 9} \\
0.6186^{*}\end{array}$ & \\
\hline $\mathrm{b}^{3} \Pi_{\mathrm{u}}$ & $\begin{array}{l}11242 \\
11256\end{array}$ & $\begin{array}{l}\mathbf{3 7 4 . 0 1} \\
373.55\end{array}$ & $\begin{array}{l}\mathbf{2 . 3 7 0} \\
2.309\end{array}$ & $\begin{array}{l}\mathbf{0 . 8 3 5 6} \\
0.8325\end{array}$ & \\
\hline
\end{tabular}

In this review, we have illustrated how laser induced fluorescence, combined with Fourier transform spectrometry has enabled us to provide a complete description of several electronic states of $\mathrm{Li}_{2}$, and to study perturbations between these states. Now that the singlet states of $\mathrm{Li}_{2}$ are well known, and excellent $a b$ initio calculations exist for the triplet states, we can pursue the study of the triplet states, in particular due to collisionally induced spectra which accompany direct laser induced fluorescence.

\section{Acknowledgements}

This work was supported by grants from NATO and from NSERC in Canada. The Bomem FT spectrometer in Lyon was financed by the CNRS, Université Lyon I and the Région Rhone Alpes.

\section{References}

1/ Schmidt-Mink, I, Müller, W., and Meyer, W., J. Chem. Phys. $\underline{80} 3311$ (1984)

2/ Konowalow D.D., and Fish J. L., Chem. Phys. $\underline{84} 463$ (1984)

3/ Spiegelmann .F, private communication

4/ Barakat B., Bacis R., Churassy S. , Field R.W., Ho J., Linton C., McDonald S., Martin F. and Vergès, J. J. Mol. Spec. 116271 (1986)

5/ Carrot F., Bacis R., Churassy S., Ho J., Linton C., McDonald S., Martin F. and Vergès J.

J. Mol. Spec. 11638 (1986)

6/ Linton C., Martin F., Bacis R. and Vergès J. J. Mol. Spec. 137235 (1989)

7/ Linton C., Martin F. and Bacis R. J. Mol. Spec. 142340 (1990)

8/ Martin F., Bacis R., Vergès J., Linton C., Bujin G., Cheng C.H. and Stad E. Spectrochim. Acta 44A 1369 (1988)

9/ Linton C., Murphy T.L., Martin F., Bacis R.and Vergès J. J. Chem. Phys. 91636 (1989)

10/ Linton C., Bacis R., Crozet P., Martin F., Ross A.J. and Vergès J. to be published. 